\title{
Magnetic Field Induced Slow Magnetic Relaxation in $\mathrm{KEr}\left(\mathrm{MoO}_{4}\right)_{2}$
}

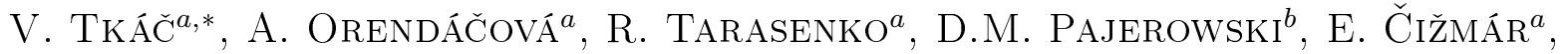 \\ M. OrendÁ $\check{C}^{a}$, A.G. Anders ${ }^{c}$, M.W. Meisel ${ }^{b}$ AND A. FeHER ${ }^{a}$ \\ ${ }^{a}$ Centre of Low Temperature Physics of P.J. Šafárik University and SAS, Faculty of Science, P.J. Šafárik University, \\ Park Angelinum 9, 04154 Košice, Slovakia \\ ${ }^{b}$ Department of Physics and the National High Magnetic Field Laboratory, University of Florida, \\ Gainesville, Florida 32611-8440, USA \\ ${ }^{c}$ B.I. Verkin Institute for Low Temperature Physics and Engineering of NASU, \\ Lenin Av. 47, 61103 Kharkov, Ukraine
}

\begin{abstract}
The spin dynamics of a layered magnetic insulator, $\mathrm{KEr}\left(\mathrm{MoO}_{4}\right)_{2}$, have been investigated in a magnetic field applied along the easy axis at temperatures where the magnetism is dominated by the occupation of a ground doublet. More specifically, the DC magnetization and AC susceptibility were studied in magnetic fields up to $5 \mathrm{~T}$ and at temperatures ranging from 1.8 to $20 \mathrm{~K}$. The temperature dependence of the AC susceptibility suggests a slowing down of magnetic relaxation with increasing magnetic field. The magnetic field dependence of the AC susceptibility, studied at nominally $2 \mathrm{~K}$, indicates the presence of low-field, intermediate-field, and highfield regimes characterized by the interplay of internal and external magnetic fields that give rise to different relaxation processes.
\end{abstract}

DOI: 10.12693/APhysPolA.127.353

PACS: $71.70 . \mathrm{Ch}, 75.30 . \mathrm{Gw}, 75.50 . \mathrm{Xx}, 75.78 .-\mathrm{n}$

\section{Introduction}

$\mathrm{KEr}\left(\mathrm{MoO}_{4}\right)_{2}$ belongs to a large group of double molybdates $\operatorname{ARE}\left(\mathrm{MoO}_{4}\right)_{2}$, where A stands for an alkali metal and RE for a rare earth. These compounds are characterized by a layered crystal structure and a strong magnetic anisotropy. Structural phase transitions resulting from a dynamic coupling of low-energy lattice vibrations with electronic excitations of $\mathrm{RE}$ ions frequently occur in these compounds [1]. A previous specific heat study identified a magnetic subsystem of $\operatorname{KEr}\left(\mathrm{MoO}_{4}\right)_{2}$ as a quasi-two-dimensional array of effective $S^{\prime}=1 / 2$ Ising chains with an intrachain ferromagnetic interaction, $\left|J_{1}\right| / k_{\mathrm{B}} \approx 0.9 \mathrm{~K}$, and an interchain antiferromagnetic coupling, $\left|J_{2}\right| \approx 0.2\left|J_{1}\right|[2]$. A phase transition to the magnetically ordered state has been observed at $T_{\mathrm{c}}=0.95 \mathrm{~K}$ in zero magnetic field [3]. The application of a magnetic field along the easy axis induces a formation of intrachain spin-cluster excitations at helium temperatures [4], and below $T_{\mathrm{c}}$, the system resembles an effective $S^{\prime}=1 / 2$ two-dimensional Ising model on a rectangular lattice with a metamagnetic transition appearing below $0.6 \mathrm{~K}$ in magnetic fields above $50 \mathrm{mT}[5]$. On the other hand, a Jahn-Teller effect induced by a magnetic field applied perpendicular to the easy axis was observed at helium temperatures $[1,6]$.

This study focuses on the spin dynamics in the magnetic field applied along the easy axis at temperatures

\footnotetext{
* corresponding author; e-mail: vladimir.tkac@student.upjs.sk
}

above the phase transition, where the contribution of spin cluster excitations to the specific heat was reported [4].

\section{Experimental details}

$\mathrm{KEr}\left(\mathrm{MoO}_{4}\right)_{2}$ is a pink, transparent and soft material that was prepared by a standard flux method at the B. Verkin Institute of Low Temperature Physics and Engineering in Kharkov. The compound crystallizes in the orthorhombic space group $P b c n\left(D_{2 h}^{14}\right)[7]$, Fig. 1. The DC magnetization and AC susceptibility

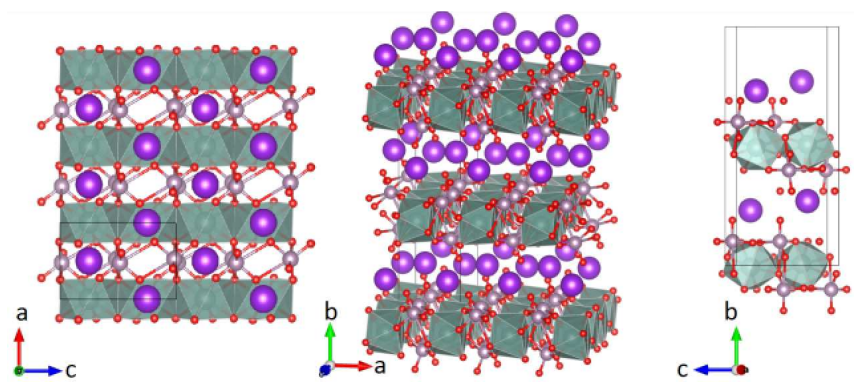

Fig. 1. The crystallographic cell of $\operatorname{KEr}\left(\mathrm{MoO}_{4}\right)_{2}$ has parameters of $a=5.063 \AA, b=18.25 \AA$ and $c=7.917 \AA$. Oxygen atoms are shown in red, potassium in purple, molybdenum in silver, and erbium in dark green with polyhedrons shown.

of $\mathrm{KEr}\left(\mathrm{MoO}_{4}\right)_{2}$ have been investigated in the temperature range from $1.8 \mathrm{~K}$ to $20 \mathrm{~K}$ in magnetic fields up to $5 \mathrm{~T}$ and frequencies from $0.1 \mathrm{~Hz}$ up to nominally $1 \mathrm{kHz}$ in a commercial Quantum Design SQUID magnetometer. A single crystal with a mass of $39 \mathrm{mg}$ was used for these magnetic measurements. The angular dependence 
of the magnetic moment was measured at $T=5 \mathrm{~K}$ and in $B=0.5 \mathrm{~T}$ using a homemade rotator [8] installed in the aforementioned commercial SQUID magnetometer.

\section{Results and discussion}

The magnetic ground state of a free $\mathrm{Er}^{3+}$ is a ${ }^{4} I_{15 / 2}$ multiplet $\left(J=15 / 2, S=3 / 2, L=6, g_{J}=\right.$ $6 / 5)$. In $\mathrm{KEr}\left(\mathrm{MoO}_{4}\right)_{2}$, the first coordination sphere of $\mathrm{Er}^{3+}$ consists of eight oxygen atoms that form a slightly distorted square antiprism, which splits ${ }^{4} I_{15 / 2}$ into 8 Kramers doublets that have energies (in K) of $E_{1}=22, E_{2}=46, E_{3}=107, E_{4}=266, E_{5}=372$, $E_{6}=456$, and $E_{7}=467$ above the ground doublet [6]. The crystallographic cell has two pairs of magnetically nonequivalent $\mathrm{Er}^{3+}$ ions with local symmetry axes tilted in the $a c$ plane by an angle of a few degrees with respect to the $a$ axis [7].

The magnetic field dependence of the DC magnetization was measured at a constant temperature of $1.8 \mathrm{~K}$, with a magnetic field applied along the $a$ axis (Fig. 2).

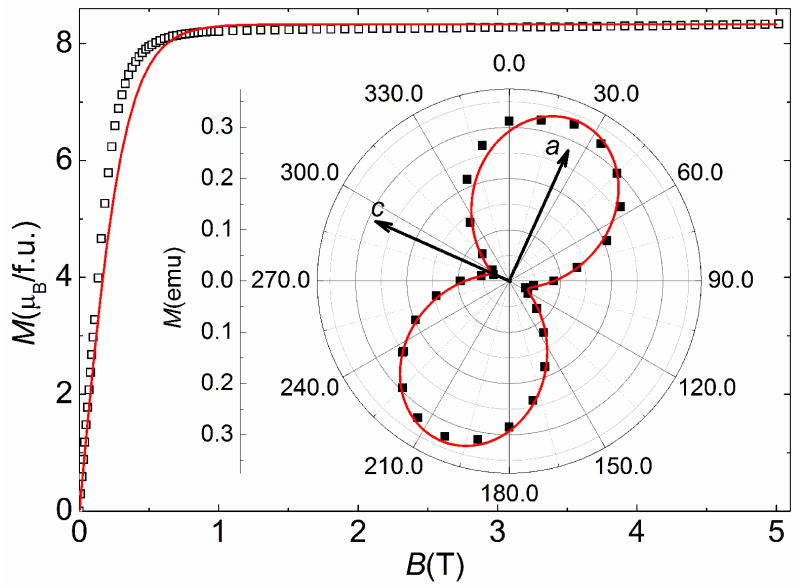

Fig. 2. The magnetic field dependence of the $\mathrm{KEr}\left(\mathrm{MoO}_{4}\right)_{2}$ magnetization (open squares) with $B \| a$, the easy axis, along with a fit to a Brillouin function (solid line) with $g=16.5$ and $S^{\prime}=1 / 2$. Inset: The angular dependence of the magnetic moment in the $a c$ plane at $T=5 \mathrm{~K}$ and in $B=0.5 \mathrm{~T}$ (full squares) is shown, along with a fit (solid line) that is described in the text.

The data were fitted to a Brillouin function with an effective spin of $S^{\prime}=1 / 2$ to yield a $g$-factor of $g_{a}=16.66$ and a saturated magnetic moment of $M_{\text {sat }}^{a}=g_{a} S^{\prime}=$ $8.33 \mu_{\mathrm{B}}$, which is close to the value associated with a pure, $J= \pm 15 / 2$ quantum state $\left(M_{\text {sat }}=g_{J} J=6 / 5 \times\right.$ $\left.15 / 2 \mu_{\mathrm{B}}=9 \mu_{\mathrm{B}}\right)$. The deviations from effective $S^{\prime}=1 / 2$ behavior are ascribed to a combination of crystal field splitting and magnetic correlations mediated by dipolar coupling.

The angular dependence of the magnetic moment measured at a constant temperature $5 \mathrm{~K}$ and magnetic field $0.5 \mathrm{~T}$ is shown in the inset of Fig. 2. The experimental data were fitted to the relation $M=M_{a c}+M_{0} \sin (\theta-$ $\left.\theta_{0}\right)$, where $M_{a c} \approx 0.19 \mathrm{emu}$ represents a median value of the magnetic moment in the $a c$ plane. $M_{0} \approx 0.15 \mathrm{emu}$ is an amplitude and $\theta_{0} \approx-21.8^{\circ}$ represents an angular displacement between the edge of the sample and the easy axis. Since the chosen temperature was too high and the magnetic field was too low for the aforementioned Jahn-Teller effect to be present, a simple relation can be used, namely $M_{a} / M_{\mathrm{c}}=g_{a} / g_{\mathrm{c}}$, where $M_{a} \approx 0.34 \mathrm{emu}$ and $M_{\mathrm{c}} \approx 0.04 \mathrm{emu}$, to obtain $g_{\mathrm{c}} \approx 1.96$. Both the large Isinglike anisotropy of the $g$-factors in the $a c$ plane as well as the closeness of the saturated moment in the $a$ direction to the pure quantum-state value indicate a weakness of a higher order anisotropy, suggesting the possibility for slow magnetic relaxation.

With this fact in mind, the temperature dependence of the AC susceptibility was studied in constant magnetic fields, $B=0 \mathrm{~T}, 0.75 \mathrm{~T}, 1.5 \mathrm{~T}, 3.5 \mathrm{~T}$ and constant frequencies $\omega /(2 \pi)=13 \mathrm{~Hz}, 133 \mathrm{~Hz}$, and $1339 \mathrm{~Hz}$ with an alternating magnetic field of $B_{0}=\mu_{0} H=0.25 \mathrm{mT}$. The real part of the AC susceptibility, $\chi^{\prime}$, is frequency independent in zero magnetic field and should be identical with the static susceptibility. The imaginary part of the AC susceptibility, $\chi^{\prime \prime}$, is negligible on the time scale of the experimental frequency window until the application of an external magnetic field (Fig. 3).

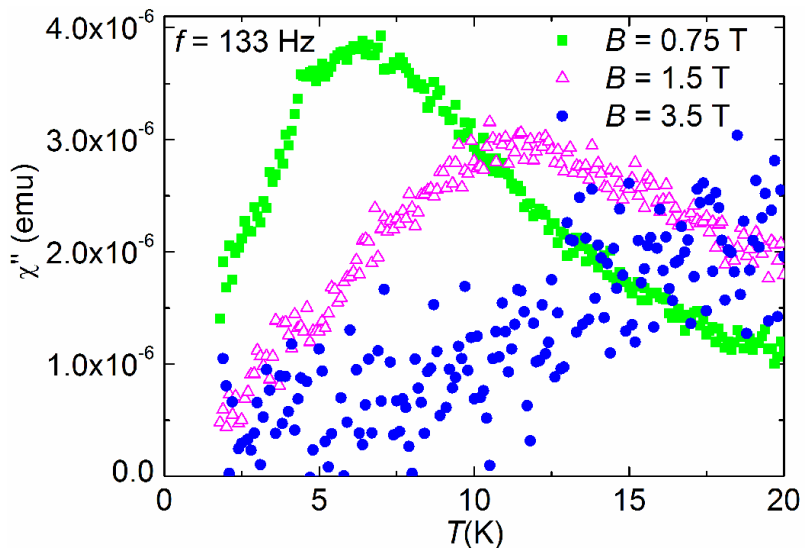

Fig. 3. The temperature dependence of the imaginary part of the AC susceptibility obtained at a frequency $f=133 \mathrm{~Hz}$ and magnetic fields $B=0.75 \mathrm{~T}, 1.5 \mathrm{~T}$ and $3.5 \mathrm{~T}$, applied along the easy axis.

The temperature dependence of $\chi^{\prime \prime}$ in a magnetic field is characterized by a single broad maximum shifting to higher temperatures with increasing magnetic field (Fig. 3). Increasing magnetic fields cause a lowering of the height of the maximum and rather severe broadening. From previous studies on a similar system $\mathrm{CsGd}\left(\mathrm{MoO}_{4}\right)_{2}$, we can conclude that the magnetic field response suggests the presence of more than one channel for the magnetic relaxation [9]. From the position of the maxima we estimated a relaxation time $\tau=1 /(2 \pi f)$, where $f$ is a measurement frequency. In magnetic fields of $0.75 \mathrm{~T}$ and $1.5 \mathrm{~T}$, the system responds with a time $\tau=1.20 \times 10^{-3} \mathrm{~S}$ at $\approx 6 \mathrm{~K}$ and $\approx 12 \mathrm{~K}$, respectively. Assuming a linear scaling of $B$ and $T$, an extrapolation of the $3.5 \mathrm{~T}$ data gives a prediction for the $\chi^{\prime \prime}$ maximum at $T \approx 28 \mathrm{~K}$. 
This simple analysis clearly indicates an important point. Specifically, when assuming a standard thermally activated relaxation process characterized by a growth of a relaxation time at lower temperatures, one can conclude the magnetic field slows-down the relaxation process, a tendency that is opposite to single-spin lattice relaxation processes. A previous study of the AC susceptibility in zero magnetic field performed at much higher frequencies, up to $140 \mathrm{MHz}$ in the temperature range from 2 to $12 \mathrm{~K}$, indicated the presence of an Orbach relaxation process, $\tau=\tau_{0} \exp \left(\Delta /\left(k_{\mathrm{B}} T\right)\right)$ with $\tau_{0}=1.31 \times 10^{-9} \mathrm{~s}$ and $\Delta / k_{\mathrm{B}} \approx 20 \mathrm{~K}$, corresponding to the energy separation between the ground state and first excited doublet [10]. This nanosecond relaxation is $10^{5}-10^{7}$ times faster than our observations under biasing magnetic fields in the same temperature range, supporting our conjecture that a magnetic field significantly slows the magnetic relaxation. Extrapolation of the aforementioned zero magnetic field Orbach relation to our frequency window would require temperatures below $2 \mathrm{~K}$. As a further step, the effect of a magnetic field on the observed slow magnetic relaxation was investigated at the lowest temperatures accessible in our setup. The magnetic field dependence of the AC susceptibility was measured at constant temperatures, $T=1.8$ and $2.1 \mathrm{~K}$ and frequencies, $\omega /(2 \pi)=\{0.13,1.3,13,133$ and 1339$\} \mathrm{Hz}$ (Fig. 4).

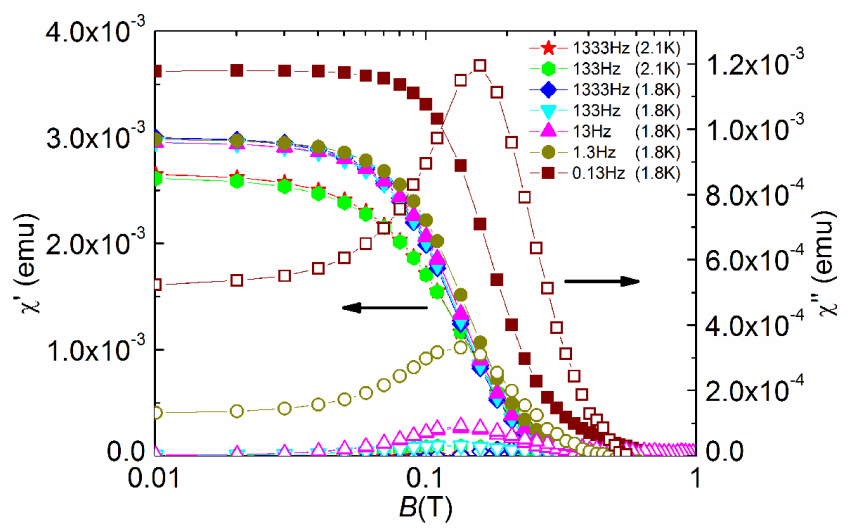

Fig. 4. The magnetic field dependence of a real part (full symbols) and imaginary part (empty symbols) of the AC susceptibility measured at different frequencies and temperatures.

A small plateau, appearing in low magnetic fields up to about $50 \mathrm{mT}$, seems to be a general feature of $\chi^{\prime}$. This field quantitatively coincides with a dipolar field of $\approx 70 \mathrm{mT}$, corresponding to the energy difference between the ground state and saturated (ferromagnetic) state, estimated in a pure dipolar approach [5]. In higher fields, $\chi^{\prime}$ and $\chi^{\prime \prime}$ gradually decrease, and nearly vanish above $B \approx 750 \mathrm{mT}$. This vanishing may be associated with the onset of a complete saturation of the DC magnetization (Fig. 2). Concerning $\chi^{\prime \prime}$, it achieves a maximum in the magnetic field, where $\chi^{\prime}$ has an inflection point (Fig. 4). Furthermore, at the lowest frequencies of $0.13 \mathrm{~Hz}$ and $1.3 \mathrm{~Hz}$, a non-zero value of $\chi^{\prime \prime}$ is indicated in $B \approx 0$, which might be connected with the aforementioned Orbach process (Fig. 4). The observed field dependence of $\chi^{\prime \prime}$ at lowest temperatures suggests the existence of separate relaxation regimes: a lowfield regime characterized by the interplay of the internal dipolar field and the external magnetic field, an intermediate-field regime accompanied by the greatest changes of DC magnetization, and finally, a high-field regime characterized by a full saturation of magnetization. Clearly, the emergence of the individual regimes depends strongly on temperature.

\section{Conclusions}

The magnetization measurements indicate the dominant influence of the crystal field generated by the first coordination sphere of $\mathrm{Er}^{3+}$ ion, which is responsible for the high magnetic anisotropy as demonstrated by the angular dependence of the magnetization in the $a c$ plane. The application of external magnetic field along the easy axis induces a magnetic relaxation that slows-down with increasing field. The field dependence of the AC susceptibility studied at about $2 \mathrm{~K}$ indicates a presence of low-field, intermediate-field, and high-field regimes characterized by the interplay of internal and external magnetic fields. In future work, the magnetic relaxation will be investigated with the aim to determine the origin of relaxation processes in the individual regions.

\section{Acknowledgments}

This work has been supported by APVV 0132-11, VEGA 1/0143/13, ITMS 26220120005, and NSF DMR0701400, DMR-1202033, and DMR-1157490 (NHMFL).

\section{References}

[1] V.I. Kutko, Low Temp. Phys. 31, 1 (2005).

[2] D. Horváth, A. Orendáčová, M. Orendáč, M. Jaščúr, B. Brutovský, A. Feher, Phys. Rev. B 60, 1167 (1999).

[3] A. Orendáčová, M. Orendáč, V. Bondarenko, A. Feher, A.G. Anders, J. Phys.: Condens. Matter 10, 1125 (1998).

[4] A.G. Anders, V.S. Bondarenko, A. Feher, A. Orendáčová, M. Orendáč, J. Magn. Magn. Mater. 187, 125 (1998).

[5] A. Orendáčová, D. Horváth, M. Orendáč, E. Čižmáč, M. Kačmár, V. Bondarenko, A.G. Anders, A. Feher, Phys. Rev. B 65, 014420 (2001).

[6] A.A. Loginov, Low. Temp. Phys. 28, 755 (2002).

[7] S. Mat’aš, E. Dudzik, R. Feyerherm, S. Gerischer, S. Klemke, K. Prokeš, A. Orendáčová, Phys. Rev. B 82, 184427 (2010).

[8] D.M. Pajerowski, M.W. Meisel, J. Phys.: Conf. Ser. 150, 012034 (2009).

[9] V. Tkáč, A. Orendáčová, R. Tarasenko, E. Čižmár, M. Orendáč, K. Tibenská, A.G. Anders, S. Gao, V. Pavlík, A. Feher, J. Phys.: Condens. Matter 25, 506001 (2013).

[10] E.N. Khatsko, A.S. Cherny, K.I. Kaplienko, Fiz. Nizk. Temp. 19, 1217 (1993). 